\title{
Positive and negative contrast effects as a function of shifts in percentage of reward
}

\author{
JEFFREY A. SEYBERT \\ University of Missouri, Kansas City, Missouri 64110
}

\begin{abstract}
Two experiments examined the effects of shifts in reward percentage on acquisition of an instrumental running response. In Experiment 1, a group shifted from 33\% reward to $100 \%$ reward ran faster following the shift than did a group that received $100 \%$ reward throughout training; that is, a positive contrast effect was observed. In Experiment 2, animals shifted from $100 \%$ to $33 \%$ reward exhibited a negative contrast effect; that is, they ran slower following the shift than did control animals that received $33 \%$ reward in both phases of the experiment. The results were discussed in terms of Capaldi's reinforcement-level theory.
\end{abstract}

Capaldi (1978) has proposed a reinforcement-level theory of instrumental learning in which he suggests that animals form expectancies in relation to the magnitude of reward that they experience. Instrumental performance is controlled, basically, as a function of the discrepancies between the animal's expectancy and what it actually receives on rewarded ( $R$ ) trials.

Recently, Seybert, Baer, Harvey, Ludwig, and Gerard (in press) have provided evidence to support Capaldi's (1978) suggestion that the reinforcement-level model might be extended to include the effects of percentage of reward. Briefly, Seybert et al. demonstrated that the often observed (e.g., Weinstock, 1958) inverse relationship between reward percentage and resistance to extinction could be most effectively explained by reinforcement-level theory.

It is clear that, in addition to explaining the effects of reward percentage on $R_{n}$, the reinforcement-level model can be extended to make predictions concerning acquisition effects of reward percentage. The experiments reported here examined two such predictions.

\section{EXPERIMENT 1}

According to the reinforcement-level model, positive contrast (i.e., faster running in a group shifted from small to large reward than in a group that always receives the large magnitude) occurs whenever an animal receives a larger reward than it expects. This discrepancy between expected and obtained reward for the upshifted animals results in a greater accumulation of habit, which elevates responding above that of nonshifted control

This research was supported in part by a grant from the Graduate Research Council, University of Missouri-Kansas City. Requests for reprints should be sent to J. A. Seybert, Department of Psychology, C. B. Annex, University of Missouri, Kansas City, Missouri 64110. animals (cf. Capaldi, 1974). Since the reinforcementlevel model has shown considerable promise in explaining extinction effects of reward percentage (Seybert et al., in press), it seems logical to assume that the model would have similar success with acquisition effects of reward percentage. Thus, we should expect the reinforcement-level model to predict a positive contrast effect based on upshifts in percentage, similar to that predicted following an upshift in magnitude of reward. The literature concerning the effects of upshifts in percentage is somewhat ambiguous. While Leung and Jensen (1968) observed positive contrast following upshifts in percentage, several authors have failed to observe such effects (e.g., Lehr, 1974, when magnitude was held constant; McCain, Lobb, \& Newberry, 1976; Roop \& Nation, 1976). Thus, the present experiment compared a group that received an upshift in reward percentage (from $33 \%$ to $100 \%$ ) to a group that received the higher percentage throughout training.

\section{Method}

Subjects. The subjects were 20 naive, male, albino SpragueDawley rats, approximately 100 days of age, purchased from the Holtzman Company.

Apparatus. The apparatus consisted of a $180 \times 16 \times 13 \mathrm{~cm}$ (length by height by width) straight-alley runway, constructed of $1.25-\mathrm{cm}$ pine, painted with alternate vertical black and white stripes $2.22 \mathrm{~cm}$ wide. The runway was divided into three sections, a $30-\mathrm{cm}$ start section, a $120-\mathrm{cm}$ run section, and a $30-\mathrm{cm}$ goal section. The sections were separated by guillotine doors and covered with hinged, hardware-cloth tops. The subject's progress in the alley was measured by three .01-sec Haydon timers. The first timer, measuring the start time, was started by activation of a microswitch at the startbox door and stopped by a photocell $15 \mathrm{~cm}$ into the run section. The second timer, measuring the run time, was activated by the first photocell and stopped by a second photocell $15 \mathrm{~cm}$ in front of the goalbox. The third timer, measuring the goal time, was activated by the second photocell and stopped by a third photocell $8 \mathrm{~cm}$ inside the goalbox. Start, run, and goal speeds were obtained by converting the respective start, run, and goal latencies to reciprocals. The sum of the start, run, and goal latencies was converted 
to a reciprocal and served as a total speed measure. This same apparatus was also used in Experiment 2.

Procedure. Upon arrival in the laboratory, the subjects were individually housed, randomly assigned to one of two groups $(\mathrm{N}=10$ /group), and placed on a diet of ad-lib food and water. Following 7 days of ad-lib food and water, the subjects were placed on a food-deprivation diet of $12 \mathrm{~g}$ of Purina Rat Chow per day. Food deprivation was initiated 7 days before the start of the experiment and continued throughout acquisition and extinction.

The two groups were designated in terms of the percentage of reward received in the preshift and postshift phases of the experiment. In the 33-100 group, animals received reward on $33 \%$ of the trials in the preshift phase, then were shifted to $100 \%$ reward (CRF) in postshift. The animals in the 100-100 group remained on a $100 \%$ reinforcement schedule in both the preshift and postshift phases. Each group received 2 days of pretraining, consisting of two reinforced running trials per day. Reinforcement, in both pretraining and acquisition training, consisted of $2 \mathrm{~cm}$ Startina Hog Starter (three pieces laid end to end; this is roughly equivalent to six $45-\mathrm{mg}$ Noyes pellets). Nonreinforcement (N) consisted of $15 \mathrm{sec}$ confinement in the unbaited goalbox.

Four different sequences of partial reinforcement (PRF) were used for the 33-100 group, as follows: Days 1 and 5, RNNRNN; Days 2 and 6, NRNRNN; Day 3, RNRNNN; Day 4, NRNNRN. For the 100-100 group, the schedule was RRRRRR for all days. The preshift portion of the experiment lasted for a period of 6 days and was followed by an 8-day postshift stage, during which both groups received six $R$ trials per day. The subjects were run in squads of six, three subjects per group in a squad, and the ITI was 5-6 $\mathrm{min}$ in both phases of the experiment. All subjects received a 20-sec delay of reinforcement on all trials in both phases of the experiment (see, e.g., Mellgren, 1972, regarding the rationale for the use of continuous delay of reinforcement in the positive contrast paradigm). The reward delay was accomplished by use of a delay guillotine door in front of the food cup, which prevented the subjects' immediate access to reward. In the preshift phase, the $33-100$ subjects received $8 \mathrm{~cm}$ (12 pieces) of Startina in the carrying cage immediately after their daily trials to equate for absolute amount of food consumed in the experimental situation.

\section{Results and Discussion}

Similar results were obtained from all sections of the alley (including the total speed measure) and, thus, only the total speed data are reported and discussed for both experiments reported here.

The data from both phases were combined in blocks of six trials (days) and may be seen in Figure 1. In the preshift phase, the 100-100 group ran faster than the 33-100 group, a relationship that was reversed following the percentage upshift on Day 7. The observations were confirmed by the results of the 2 (groups) by 14 (days) analysis of variance conducted on the data. Although the groups main effect was not significant $(F<1)$, the blocks main effect $[F(13,234)=74.95, p<.001]$ and the Groups by Blocks interaction $[\mathrm{F}(13,234)=5.04$, $\mathrm{p}<.001]$ were highly significant. Subsequent comparisons (the comparisons reported for Experiments 1 and 2 were $t$ tests conducted at the .05 level of significance) indicated that Group 100-100 was superior to Group 33-100 during preshift (Days 3 and 4 combined) $[\mathrm{t}(18)=2.62]$, and that the reverse was true during postshift (Days 2-5 combined) $[\mathrm{t}(18)=2.24]$; that is,

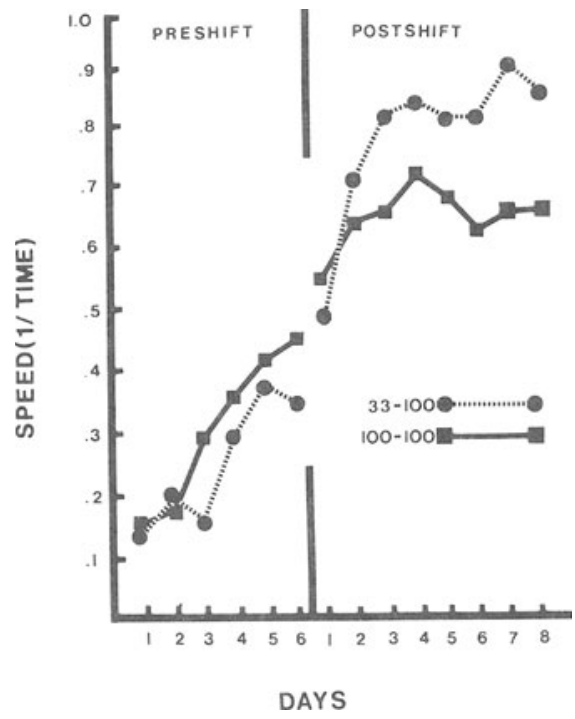

Figure 1. Mean total speeds in the preshift and postshift phases for the upshifted group (33-100) and the control group $(100-100)$ in Experiment 1.

a reliable positive contrast effect was observed following an upshift in reward percentage. The reinforcementlevel view predicts a larger positive contrast effect with delay of reward, because, according to Capaldi (1978), effective habit strength is smaller under delayed than under immediate reward. The smaller the effective habit strength, the greater will be the increase in running speed that results from an increment in habit, such as that which accompanies an upshift in reward. The present positive contrast effect resulting from an upshift in reward percentage supports this view (at least in regard to delayed reward).

A number of interesting features of the data should be noted. The superiority of the larger percentage in preshift is similar to the McCain et al. (1976) finding that $75 \%$ reward generated faster responding than $25 \%$ reward, at least early in training. Although it is not possible to determine, either from the present data or from the McCain et al. results, whether differential percentage would result in differential asymptotic performance, these data do indicate that percentage will influence rate of approach to asymptote. The absence of a partial reinforcement acquisition effect (faster running by PRF animals than by CRF animals in certain alley sections later in training) is due, presumably, to the relatively limited number of acquisition trials (36) in the present experiment. Also, the positive contrast observed in the present experiment seems to be relatively durable, the differences remaining large through 48 trials of postshift with no indication that they might diminish. This result is in agreement with a similar finding (Mellgren, Seybert, Wrather, \& Dyck, 1973) from a reward-shift procedure and also is predicted by the reinforcement-level model (for reward magnitude 
contrast) because of greater habit in the unshifted group than in the control group (even though reward expectancy in the upshifted group should eventually approximate that in the control, the greater habit generated by the increase in expectancy following the shift should not dissipate). This finding further supports the previously discussed extension of the reinforcement-level model to the present percentage findings.

The reasons for the discrepancy between the present findings and those of previously mentioned studies that failed to observe positive contrast following upshifts in reward percentage (Lehr, 1974; McCain et al., 1976; Roop \& Nation, 1976) are unclear. It should be noted, however, that several procedural differences exist between those reports and the present experiment. For example, the Lehr study utilized an operant conditioning paradigm, and the McCain et al. and Roop and Nation studies did not use a delayed reward procedure. It is possible that the discrepant findings are due to such procedural differences.

\section{EXPERIMENT 2}

Negative contrast, that is, slower running in a group shifted from large to small reward than in a group that always receives the small reward, occurs, according to the reinforcement-level model, whenever an animal receives a smaller reward than it expects. The discrepancy between expected and obtained reward for the downshifted animals results in an accrual of inhibition that, in turn, leads to depressed responding (cf. Capaldi, 1974). According to the present extension of the reinforcement-level model, a concomitant negative contrast effect would be expected if percentage of reward was downshifted, rather than magnitude. As with positive percentage contrast, the data concerning the effects of downshifts in percentage are somewhat ambiguous. McCain et al. (1976) observed a negative contrast effect following a downshift in percentage (albeit, the third shift in a repeated-shift paradigm). However, McHose and Peters (1975) and Mikulka, Lehr, and Pavlik (1967) failed to observe negative percentage contrast when only percentage was downshifted (i.e., when reward magnitude was maintained at the preshift level). Thus, the present experiment compared a group that received a downshift in reward percentage (from $100 \%$ to $33 \%$ ) to a group that received the lower percentage throughout training.

\section{Method}

Subjects. The subjects were 20 male albino rats exactly like those used in Experiment 1.

Procedure. The housing, maintenance, deprivation, and pretraining procedures were identical to those in Experiment 1. Reinforcement and nonreinforcement were the same, with one exception: The subjects did not receive a delay of reinforcement during either phase of the experiment. At the start of training, the subjects were randomly assigned to one of two groups ( $N=10$ /group). The two groups were designated in terms of the reward percentage received in the preshift and postshift phases of the experiment. In the 100-33 group, animals received reward on $100 \%$ of the trials in the preshift phase, then were shifted to $33 \%$ in postshift. The animals in the 33-33 group remained on $33 \%$ reward in both phases. The control (33-33) group received six trials per day, arranged in the following repeating PRF sequences: Day 1, RNNRNN; Day 2, RNRNNN; Day 3, NRNRNN; Day 4, NRNNRN. These schedules were repeated as necessary over a total of 13 days (during both the preshift and postshift phases). The shifted group (100-33) received six trials per day on a CRF schedule for Days 1-5. On Day 6, these animals were shifted to the above $33 \%$ PRF schedule. The 100-33 group received the complete 4-day sequence twice over the 8 days of postshift. The squad arrangements and ITI were identical to those employed in Experiment 1 . In addition, adjustments in food consumed (similar to those used in the previous experiment) were made in the carrying cage following completion of the daily trials for both groups when they were receiving $33 \%$ reward.

\section{Results and Discussion}

As in Experiment 1, the data from both phases were combined in blocks of six trials (days) for purposes of analysis. The data from Experiment 2 may be seen in Figure 2. In the preshift phase, the 100-33 group ran faster than the 33-33 group, and the reverse relationship was observed in the postshift phase. The results of the 2 (groups) by 13 (days) analysis of the data confirmed these impressions. Once again, the groups main effect was not significant $(\mathrm{F}<1)$, whereas the blocks main effect $[F(12,216)=41.48, p<.001]$ and the Groups by Blocks interaction $[F(12,216)=4.29, p<.001]$ were highly significant. Subsequent comparisons indicated that 100-33 was superior to 33-33 during preshift (Days 3 and 4 combined) $[\mathrm{t}(18)=2.34]$, and that the reverse was true during postshift (Days 2-5 combined) $[t(18)=1.81]$; that is, a reliable negative contrast effect was observed following a downshift in reward percent-

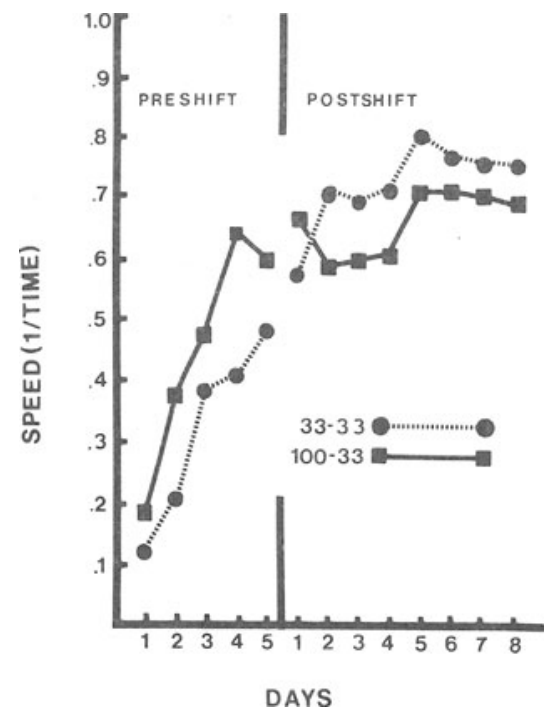

Figure 2. Mean total speeds in the preshift and postshift phases for the downshifted group $(100-33)$ and the control group (33-33) in Experiment 2. 
age. As in Experiment 1, the reasons for the discrepancy between the present negative contrast findings and the results of studies that have failed to observe negative contrast following downshifts in percentage (McHose \& Peters, 1975; Mikulka et al., 1967) are unclear. Once again, the superiority of the larger percentage in preshift replicates a similar finding by McCain et al. (1976). Although the negative contrast effect observed here was relatively durable (the differences were stable for approximately 36 trials), there are indications that the effect may have been dissipating when the postshift phase was terminated. This reduction in the differences is predicted from the reinforcement-level model (for reductions in reward magnitude) because, according to Capaldi (1978), a reward reduction is accompanied by a discrepancy between obtained and expected reward. Such a discrepancy results in an increase in conditioned inhibition (which will presumably lead to a relatively permanent depression of responding), and also occasions fading (frustrative) traces. The fading traces will presumably dissipate as expected reward more closely approximates obtained reward, since the obtainedexpected discrepancy itself will be gradually reduced, all of which should lead to some diminution of the negative contrast effect, as was observed here.

\section{GENERAL DISCUSSION}

The present experiments demonstrated that shifts in reward percentage during acquisition produce both positive and negative contrast effects similar to those observed following analogous shifts in reward magnitude (e.g., Mellgren, 1972). Specifically, in Experiment 1, animals shifted from $33 \%$ to $100 \%$ reward ran faster following the shift than did animals that always received $100 \%$ reward (positive contrast). Similarly, in Experiment 2, subjects shifted from $100 \%$ to $33 \%$ reward ran slower following the shift than did subjects receiving 33\% reward in both training phases (negative contrast). These results were explained using Capaldi's (1978) reinforcement-level model. Briefly, that model holds that instrumental responding is controlled, in large measure, in both acquisition and extinction by discrepancies between expected and obtained reward magnitude. We believe that the present data strongly support the contention that the reinforcement-level model can explain the effects of percentage of reward, as well as those of reward magnitude. That is, we are suggesting that frequency of reward, as well as discrepancies between expected and obtained frequency of reward, can also contribute to the animal's overall reward expectancy and thereby exert powerful control over instrumental responding. The present findings, together with those of Seybert et al. (in press), provide strong support for such a suggestion.

\section{REFERENCES}

Capaldi, E. J. Partial reward either following or preceding consistent reward: A case of reinforcement level. Journal of Experimental Psychology, 1974, 102, 954-962.

CAPAldi, E. J. Reinforcement level: An expectancy-associative approach to relative reinforcement and nonreinforcement effects. In J. Baerwaldt (Ed.), The Arlington symposium on learning. Stanford, Conn: Greylock, 1978.

Lehr, R. Partial reward and positive contrast effects. Animal Learning \& Behavior, 1974, 2, 221-224.

LeUng, C. M., \& Jensen, G. D. Shifts in percentage of reinforcement viewed as changes in incentive. Journal of Experimental Psychology, 1968, 76, 291-296.

McCaIN, G., LobB, M., \& Newberry, J. Extended training and multiple shifts: Percentage of reward. Bulletin of the Psychonomic Society, 1976, 8, 191-193.

Mchose, J. H., \& Peters, D. P. Partial reward, the negative contrast effect, and incentive averaging. Animal Learning \& Behavior, 1975, 3, 239-244.

Mellgren, R. L. Positive and negative contrast effects using delayed reinforcement. Learning and Motivation, 1972, 3, 185-193.

Mellgren, R. L., Seybert, J. A., Wrather, D. M., \& Dyck, D. G. Preshift reward magnitude and positive contrast in the rat. American Journal of Psychology, 1973, 86, 383-387.

Mikulka, P. J., Lehr, R., \& Pavlik, W. B. Effects of reinforcement schedules on reward shifts. Journal of Experimental Psychology, 1967, 74, 57-61.

Roop, S. S., \& Nation, J. R. Positive contrast following a shift from partial punishment to continuous reinforcement. Psychological Reports, 1976, 39, 934.

Seybert, J. A., Baer, L. P., Harvey, R. J., Ludwig, K., \& GERARD, I. C. Resistance to extinction as a function of percentage of reward: A reinforcement level interpretation. Animal Learning \& Behavior, in press.

Weinstock, S. Acquisition and extinction of a partially reinforced running response at a 24 -hour intertrial interval. Journal of Experimental Psychology, 1958, 56, 151-158.

(Received for publication September 29, 1978.) 